\title{
Burnout symptoms common among medical residents but rates vary across specialties
}

\author{
n Cite as: CMAJ 2018 October 15;190:E1239. doi: 10.1503/cmaj.109-5664
}

Posted on cmajnews.com on Sept. 27, 2018.

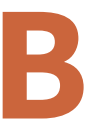

urnout has become a growing concern in the medical profession, and it appears to be a problem that starts early in a physician's career. According to a recent study, almost half of medical residents report symptoms of burnout.

The study, published in the Journal of the American Medical Association (JAMA), involved 3588 second-year medical residents in the United States. Nearly half $(45.2 \%)$ reported at least one symptom of burnout, such as emotional exhaustion or depersonalization. About one in seven (14.1\%) expressed regret about pursuing medicine as a career.

However, there was a wide range of rates reported for both measures across clinical specialties. Rates of burnout symptoms ranged from $29.6 \%$ to $63.8 \%$, and the range for career-choice regret was $7.4 \%$ to $32.7 \%$. The specialties with the highest reported rates of burnout were urology $(63.8 \%)$, neurology $(61.6 \%)$ and ophthalmology (55.8\%).

"These findings suggest the increased burnout among physicians in these specialties may be attributable, in part, to unique characteristics of the work intrinsic to these specialties," according to the researchers. "Alternatively, the high prevalence of burnout symptoms among supervising physicians in these specialties may adversely affect the learning environment, or these supervising physicians may model burnout to resident physicians, placing the resident physicians who are training in these specialties at greater risk."

Dermatology residents were the least likely to report symptoms of burnout (29.6\%). Primary care specialties fell somewhere in the middle of the pack: $37.2 \%$ for

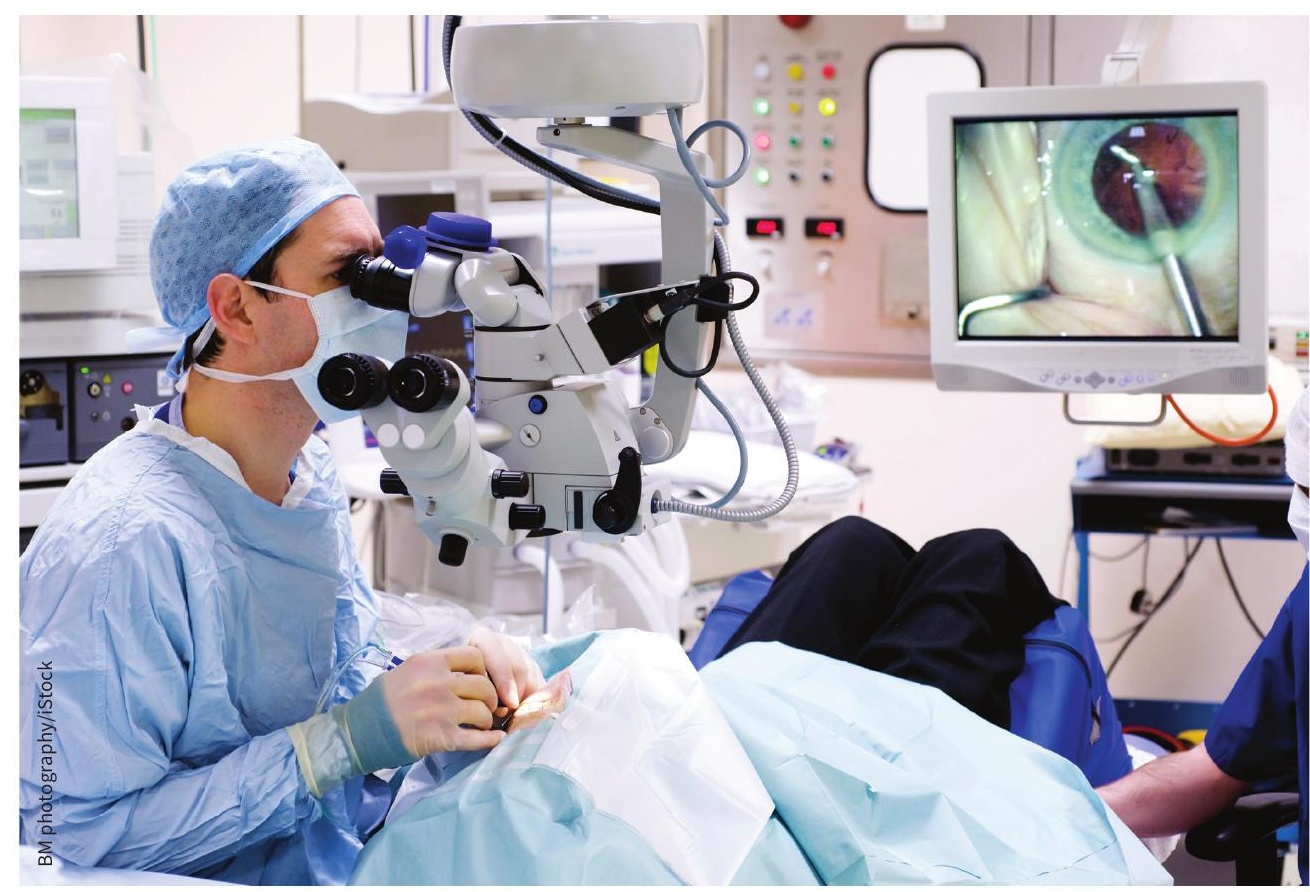

Medical residents in some specialties, including ophthalmology, report high rates of burnout symptoms.

family medicine, $42.6 \%$ for internal medicine and $43.2 \%$ for pediatrics.

Residents who reported high levels of anxiety during medical school, and low levels of empathy, were found to be at higher risk of burnout during residency. Also, female residents were more likely to report signs of burnout, as were respondents with children and those of Hispanic or Latino ethnicity. Overall, residents who reported signs of burnout were 3.2 times more likely to report regret about entering medicine.

An editorial accompanying the study, however, cautions about jumping to conclusions about the extent of the problem of physician burnout. The authors question the reliability of existing data on the subject and suggest it is inappropriate to simply accept the often-quoted statement that half of doctors are burned out, calling for more study on the matter.

"The term burnout has taken on meaning far beyond what is understood about it as an actual diagnosis or even a syndrome. The medical profession has taken a self-reported complaint of unhappiness and dissatisfaction and turned it into a call for action on what is claimed to be a national epidemic that purportedly affects half to two-thirds of practising physicians," state the editorial's authors. "If a construct like burnout is so variable in its prevalence, then either its measures are highly unreliable, or seemingly similar populations differ widely in risk. Both explanations are worthy of study."

Roger Collier, CMAJ 